\title{
Particularity of Content and Illusions of Identity
}

\author{
Błażej Skrzypulec ${ }^{1}$
}

Received: 13 February 2018 / Accepted: 26 April 2018 / Published online: 7 May 2018 (C) The Author(s) 2018

\begin{abstract}
This paper argues that the accuracy of perceptual experiences cannot be properly characterized by using the particular notion of content without breaking one of the three plausible assumptions. On the other hand, the general notion of content is not threatened by this problem. The first assumption is that all elements of content determine the accuracy conditions of an experience. The second states that objects needed for the accuracy of experiences are physical entities that stand in a perceptual relation to a subject. According to the third assumption, common experiences do not have accuracy conditions that are impossible to satisfy. The above point is demonstrated by analysing illusions of identity in which the number of objects is represented incorrectly. In the concluding parts of the paper, I investigate how an alternative account of particular content can be developed by rejecting the first assumption.
\end{abstract}

Keywords Perception · Illusions · Hallucinations · General content · Particular content $\cdot$ Accuracy

The notion of "representational content" is one of the crucial concepts in contemporary debates concerning perceptual experiences. It is used to express the intuition that perceptual experiences present the world as being in the certain way and in doing so they may be accurate or inaccurate (Bach 2007; Byrne 2001; Byrne and Logue 2008; Chalmers 2006; Kriegel 2009; Schellenberg 2011). For instance, if one has an experience as of a red square, then a red square is a content of one's experience, and without the presence of such a square the experience would not be

Błażej Skrzypulec

blazej.skrzypulec@gmail.com

1 Institute of Philosophy and Sociology, Polish Academy of Science, Warsaw, Poland 
accurate. For this reason, it is common to characterize representational content as determining the accuracy conditions of perceptual experiences, or even as being identical to their accuracy conditions (Schellenberg 2011; Siegel 2010).

Philosophers of perception disagree on whether the notion of "representational content" is needed to adequately characterize perceptual experiences (Brewer 2006; Genone 2014; Schellenberg 2010; Travis 2014). However, in this paper I address a more specific issue that concerns those positions in which it is accepted that perceptual experiences have content: a distinction between general and particular content (Martin 2002; Prat 2006; Schellenberg 2016; Soteriou 2000). According to the general content view, the representational content specifies an object that has certain features, for instance a red square. In contrast, in the case of particular content, what is specified is not an object with certain features, but the particular object: this particular red square and not an indefinite red square.

In recent years many authors have developed arguments aimed to show that the particular notion of content has significant advantages over the general notion. These arguments can be divided into three general types. First, it is claimed that general content is implausible given the phenomenology of perceptual experiences (Schellenberg 2016; Martin 2002). Second, it has been proposed that general content cannot account for higher-order abilities based on perceptual experiences, such as forming singular thoughts (Brewer 2015; Nanay 2012). Third, it is argued that the general notion of content cannot properly attribute accuracy to certain perceptual experiences, i.e., it entails that some intuitively accurate experiences are inaccurate or vice versa (Dokic 1998; Schroer 2014).

In this paper, I focus on arguments concerning the attribution of accuracy, and I argue for a different conclusion-that the general notion has advantages over the particular notion. More specifically, I claim that the particular notion of content cannot properly attribute accuracy to perceptual experiences given the three following assumptions: (A) every element of content contributes to the determination of accuracy conditions; (B) entities whose presence is needed for the accuracy of perceptual experiences are physical, external entities that stand in a proper perceptual relation to a subject; and (C) usual perceptual experiences do not have deviant contents that make experiences necessarily inaccurate. I show that this difficulty is apparent in cases of illusions of identity, i.e., illusions in which one perceives more objects or fewer objects than there really are. This constitutes an important problem for the particular notion of content as $(\mathrm{A})-(\mathrm{C})$ are intuitive constraints. However, in the final parts of the paper, I show how proponents of particular content can develop an alternative account of content by rejecting the assumption (A).

The paper starts by explicating assumptions (A)-(C) (Sect. 1). Subsequently, it is investigated how by using the general and particular notions of content one can characterize the accuracy of veridical perceptions, illusions, and hallucinations (Sect. 2), as well as illusions of identity (Sect. 3). I argue that only by using the general notion of content can the accuracy of identity illusions be properly characterized without breaking one of the assumptions (A)-(C). Section 4 discusses various solutions that can be applied by a proponent of particular content to solve the considered problem. 


\section{Three Assumptions}

By "general content" I mean content that can be properly described using quantified general formulas of predicate logic without the use of constants or predicates that express the individual nature of objects (like "being the object $a$ "). For instance, a general content connected with an experience as of a red object can be represented by a formula $\exists_{x} R(x)$ where the predicate $\mathrm{R}$ designates the property of redness. Analogously, the particular content is such that it can be properly expressed by formulas of predicate logic containing constants. In this case, the content associated with a veridical experience as of a red object would be $R(a)$ where $a$ is the particular object that is perceived by a subject. Constants can be interpreted as proper names denoting particulars, or as demonstrative terms like this or that designating particular entities to which a subject is perceptually related (Almäng 2013; Brewer 2006).

While I use the terminology of attributing properties to objects, nothing in the presented argumentation relies on whether one accepts a Russellian or a Fregean theory of perceptual content (Chalmers 2006; Schellenberg 2011). From the Russellian perspective, the formula $R(a)$ is interpreted as describing the object $a$ as having the property of redness, while from the Fragean perspective the same formula characterizes the object $a$ under the red mode of presentation or as distinguished by applying the concept of redness. Furthermore, I only consider whether particular objects figure in content, and my argumentation does not address the issue of whether perceptually experienced properties are universals or tropes (Almäng 2013; Mulligan 1999; Nanay 2012). In addition, I consider so-called 'self-referential' contents, which include a reference to the experience or the subject itself, as general contents because their particular elements are not objects that are perceived by a subject (Burge 1991; Prat 2006).

I argue that the particular notion of content does not properly ascribe accuracy to perceptual experiences, i.e., it entails that intuitively accurate experiences are inaccurate, or that intuitively inaccurate experiences are accurate, given that three plausible assumptions are accepted.

The first assumption (A) is that every element of content contributes to the determination of the accuracy conditions of an experience. For instance, in the case of a general content $\exists_{x} R(x)$, both the quantified variable $x$ and the predicate $R$ determine the accuracy conditions by specifying that for an experience to be accurate, an object must be present, and it must possess the property designated by $R$. Analogously, in the case of particular content $R(a)$, the constant $a$ determines that the presence of the particular object $a$ is needed for accuracy, and the predicate $R$ determines that this object must possess the property designated by $R$.

This assumption is commonly accepted in contemporary characterizations of perceptual content and its relevance can be demonstrated by utilizing cases of so-called 'veridical illusions' (Soteriou 2000). Let's imagine a situation in which a person has an experience as of an object in place $P$. However, it is only a mirror image created by the object $a$ positioned outside the visual field in place $L$. Nevertheless, just behind the mirror there is a distinct object $b$ positioned in $P$. Such an experience is intuitively inaccurate despite the fact that there really is an object in place $P$. 
It is often claimed that the particular notion of content has the advantage of being able to easily characterize such veridical illusions as inaccurate experiences (Dokic 1998; Prat 2006; Schellenberg 2016; Schroer 2014; Soteriou 2000). This can happen exactly because all elements of content determine the accuracy conditions. In the above example, the particular content is $P(a)$ where the element $P$ designates the property of being located in $P$ and $a$ is the object to which a person is, in fact, perceptually related, i.e., the object that creates the mirror image. These two elements jointly determine that the considered experience would be accurate if, and only if, the object $a$ is in $P$ (or, using Fregean terminology, is such that it is suitable for being presented under the $P$ mode of presentation). From this we can infer that the experience is illusory, as the object positioned in $P$ is the object $b$ and not $a$.

According to the second assumption (B), objects that are necessary for the accuracy of perceptual experiences are external, physical objects that stand in an appropriate perceptual relation to a subject having this experience. This assumption contains two claims. First is that objects that are necessary for accuracy have to be connected with a subject by a perceptual relation probably involving, inter alia, a causal link between an object's surface and perceptual mechanisms (Almäng 2013; Byrne 2001; Prat 2006; Soteriou 2000). The theoretical importance of this claim can again be demonstrated by referring to the veridical illusion case above. For the accuracy of an experience it is not enough that there exists an object $b$ in place $P$ if this object is not linked with a subject in such a way that we can state that the subject perceives $b$. The second claim is that that objects that are necessary for accuracy are physical, external entities, and therefore not mental entities. Treating them as mental entities would result in a position that is a version of the sense-data theory. Given the amount of serious difficulties that sense-data theories face, such a consequence is generally avoided in contemporary philosophy of perception (Schellenberg 2010, 2011).

The third assumption (C) is that the contents of usual perceptual experiences should not be deviant contents that provide accuracy conditions that cannot be fulfilled. There may be special types of illusions, like a waterfall illusion where something seems to be both moving and not moving, whose content is self-contradictory (Crane 1988). Furthermore, hallucinations are so detached from ordinary perceptual experiences that it may be justified to characterize them as having content with accuracy conditions that cannot be met (Schellenberg 2010). However, accurate perceptual experiences and the majority of illusions seem to present some possible ways in which the environment could be, and so it is implausible that their content determines unsatisfiable accuracy conditions.

In the subsequent sections, I investigate how the general and the particular notions of content attribute accuracy to various perceptual experiences given the three assumptions (A)-(C) described above. I argue that experiences involving an illusion of identity show that only the general account of content allows for proper accuracy attribution. 


\section{Perceptions, Illusions and Hallucinations}

Let's consider a perceptual experience presenting a red object on the left and a green object on the right. If this experience is an accurate perception, then in fact, there is such a red object on the left and such a green object on the right, connected with a subject by perceptual relations. Using the general notion of content, the content of the considered experience can be expressed by the following quantified formula (for short, $\mathrm{R}$ designates being red and on the left and $G$ represents green and on the right):

$$
\exists_{x, y} R(x) \wedge G(y) \wedge x \neq y
$$

This content determines that the experience is accurate if, and only if, there are two objects, one with the properties designated by $R$ and the second with the properties designated by $G$. Given this, the content (1) properly characterizes the considered experience as accurate in a situation in which one is, in fact, perceptually related to a red object on the left and to a green object on the right.

According to the particular notion of content, the content would not be expressed by a quantified formula, but instead, by a formula with two constants:

$$
R(a) \wedge G(b)
$$

Such content determines that the experience is accurate if, and only if, there are two objects, $a$ and $b$, such that $a$ has properties designated by $R$, while $b$ has properties designated by $G$. The dominant view on particular content is that the objects that figure in content are those that stand in a perceptual relation to a subject. Given this, this notion of content also properly characterizes the considered experience as accurate in a situation in which one is perceptually related to a red object on the left, and a green object on the right.

Of course, the considered experience does not have to be accurate. For instance, it can be illusory because the object on the left is in fact yellow and not red. However, in such a situation, both the general and particular accounts of content correctly ascribe accuracy to the considered experience. The accuracy conditions specified by the general content (1) are not satisfied, as there is no object with the properties designated by $R$. This is also the case with accuracy conditions determined by the particular content (2). These conditions are not satisfied because the object $a$ has properties different to those designated by $R$.

The situation is more complex for a proponent of particular content if the considered experience is hallucinatory as, for instance, not only does the environment not contain a red object on the left and a red object on the right, it in fact does not contain any objects to which a subject is perceptually related. The general content (1) properly characterizes such a hallucinatory experience as inaccurate because there are not two objects with appropriate characteristics in the hallucinatory case. On the other hand, a proponent of the particular notion of content cannot use (2) as the content of the hallucinatory experience, because the content (2) contains particular objects, $a$ and $b$, to which a subject is perceptually related. However, there are no such objects in the hallucinatory case. One influential idea 
is to explain hallucinatory cases by referring to so-called 'gappy content' that lacks particular objects (Schellenberg 2010, 2011, 2016):

$$
R\left(\_\right) \wedge G\left(\_\right)
$$

Gappy content describes a situation in which properties are attributed but there are no objects that can be their bearers, as there are no objects perceptually related to a subject. It is claimed that such content entails accuracy conditions that cannot be fulfilled, for the reason that the accuracy presupposes that there is an object to which a subject is related. As a result, all hallucinatory experiences are necessarily inaccurate (Schellenberg 2010, p. 40).

The above considerations show that both the general and particular view on content can properly ascribe accuracy to typical accurate perceptions, illusions, and hallucinations. Furthermoer, they are able to do so without breaking the three assumptions (A)-(C). First, in contents (1)-(3) all elements contribute to the determination of the accuracy conditions. Second, the objects necessary for accuracy are not mental entities but physical objects to which a subject is perceptually related. Third, the only cases in which content determines accuracy conditions that cannot be satisfied are hallucinatory experiences, as described from the particular content perspective. However, such a move seems to be justified, as hallucinations are nonstandard experiences that are plausible candidates for having a deviant content.

Apart from 'standard' illusions and hallucinations, philosophers of perception also discuss special cases of 'veridical hallucinations' and 'veridical illusions', as it is often believed that these pose an additional problem for general accounts of content. Let's consider a rather unlikely case in which one has a perceptual experience as of a red square, a red square is really present in a proper place, but a subject is not appropriately related to it. For instance, this may happen because the neural activity underlying the considered experience is not caused by light reflected from the square but is generated by a psychoactive substance. It seems intuitive that due to a lack of the proper perceptual relation the experience is inaccurate.

Such 'veridical hallucinations' are believed to pose a problem for the general notion of content (Prat 2006) because if the content of the above veridical hallucination is $\exists_{x} R(x)$, where $R$ designates the properties of a square, then accuracy conditions are satisfied. Therefore, the veridical hallucination is, contrary to the intuition, characterized as an accurate experience; general content seems to be insensitive to the occurrence of perceptual relations. On the other hand, the particular notion of content does not face such a problem. In the considered veridical hallucination a subject does not stand in a perceptual relation to any object, and so the content would be gappy: $R\left({ }_{-}\right)$. Of course, this renders a veridical hallucination as an inaccurate experience.

An analogous observation is made when veridical illusions are considered, i.e., experiences that attribute features that are really instantiated but are not instantiated by objects to which a subject stands in a perceptual relation (Soteriou 2000). Let's examine again a veridical illusion from Sect. 1. In this case, one has an experience as of an object in place $P$. What is really present is a mirror image of an object in $P$ caused by the object $b$ that is positioned outside the visual field. Nevertheless, just behind the mirror there is another object $a$ located in $P$. 
The particular notion of content can easily characterize such veridical illusion as inaccurate. In this case the content would be $P(b)$, where $P$ designates the locationproperty and $b$ is the particular to which a subject is, in fact, perceptually related, i.e., the object that generates the mirror image. The accuracy conditions determined by this content are not satisfied as it is, in fact, object $a$ and not $b$ which is in place $P$. On the other hand, it seems that the same result cannot be obtained by using the general notion of content. If the general content of the considered veridical illusion is $\exists_{x} P(x)$, then this illusory experience is counterintuitively accurate because there is, in fact, an object in $P$, i.e., the object $a$.

A popular way to amend this problem is to extend the general content, such that it encompasses the occurrence of perceptual relations (see Burge 1991; Almäng 2013; Schroer 2014; Searle 1983 for variants). Such notions of content are often called self-referential, since according to them, a part of the content specifies a relation between objects and the experience (or the perceptual system, or the subject) itself. For instance, this relation can be characterised as a causal link or as a form of noncausal acquaintance. A simple version of such an extended general content in the considered case of veridical illusion is as follows:

$$
\exists_{x} P(x) \wedge \operatorname{REL}(x)
$$

In (4) the predicate REL designates the occurrence of a perceptual relation between an object and the subject. In consequence, the content (4) determines that the experience is accurate if, and only if, there is an object in place $P$ that stands in a perceptual relation to the subject. In this case, the veridical hallucination is correctly characterized as inaccurate because while there is an object in $P$, it is not perceptually related to the subject.

A potential disadvantage of such a proposal is that it introduces elements of content that are not accessible to a subject, due to not figuring in the phenomenal character of an experience (see Dokic 1998; Pereira 2016; Prat 2006). It may be argued that while we have phenomenal access to properties such as squareness or redness, this is not the case with the occurrence of perceptual relations, like causal or acquaintance relations, between objects and a subject. As a result, proponents of the extended versions of general content seem to be forced to make a distinction between internal content that is phenomenally accessible and external content that is not.

Nevertheless, while the above consequence may be controversial, it does not force proponents of the general content to abandon any of the assumptions (A)-(C). This is because including relations within content does not change-even in case of 'veridical hallucinations' and 'veridical illusions' - the fact that all elements of general content contribute to specifying the accuracy conditions, accuracy demands external objects standing in perceptual relations, and it is possible to satisfy specified accuracy conditions. Because my goal is to assess the consistency of the general and particular notions of content with assumptions (A)-(C), and not to investigate other potential problems of these accounts, I will discuss the advantages and disadvantages of introducing relations within content no further.

Overall, it seems that both general and particular notions of content are capable of properly attributing accuracy to hallucinations and illusions, while satisfying 
assumptions (A)-(C). In doing so, the particular notion must adopt a special treatment of hallucinatory cases, both 'standard' and 'veridical', while the general notion must introduce perceptual relations within content in order to properly describe veridical hallucinations and illusions. Despite the fact that these theoretical moves may be viewed as controversial for various reasons, both notions of content succeed in satisfying assumptions (A)-(C). First, all elements of content contribute to determining the accuracy conditions. Second, the objects needed for the accuracy of experiences are physical objects standing in perceptual relations to a subject. Third, only veridical hallucinations described in accordance with the particular notion of content have deviant content, but this is justified by their special status. In the next section, I argue that illusions of identity constitute an exception: only the general notion of content can accurately characterise their accuracy conditions while maintaining the three above assumptions.

\section{Illusions of Identity}

Illusions of identity fall into two categories. First, where an experience represents fewer objects than there really are. Second, where the number of represented objects is higher than the actual number of objects. Probably the most common example of the first type is the apparent motion phenomenon, which consists of experiencing a series of differing particulars as one single changing object, due to the occurrence of appropriate spatiotemporal relations. A popular psychological demonstration of apparent motion is the phi-phenomenon, where two alternatively appearing and disappearing light-spots are experienced as one moving object (e.g., Matthen 2004).

Let's consider a minimal case of apparent motion in which one has an experience as of an object in place $P 1$ at moment $T 1$, and an experience as of the same object in place $P 2$ at a subsequent moment $T 2$. However, in fact there are two objects: $a$ in $P 1$ at $T 1$ and $b$ in $P 2$ at $T 2$, and therefore the considered experience is illusory and should be classified as inaccurate.

The accuracy of the apparent motion illusion can be easily characterized using the general notion of content. In the considered case, the content would be as follows ( $P 1 T 1$ designates the property of being in $P 1$ at $T 1$ while $P 2 T 2$ designates the property of being in $P 2$ at $T 2)$ :

$$
\exists_{x, y} P 1 T 1(x) \wedge R E L(x) \wedge P 2 T 2(y) \wedge R E L(y) \wedge x=y
$$

The content (5) determines that the experience is accurate if, and only if, there is an object in $P 1$ at $T 1$ that is perceptually related to the subject, and there is an object in $P 2$ at $T 2$ that is perceptually related to the subject, and the object at $T 1$ is the same as the object at $T 2$. This renders the apparent motion experience inaccurate because, in fact, a subject is perceptually related to two and not to one object.

However, the analogous effect cannot be obtained by applying the particular notion of content. In particular content the elements of content are objects to which a subject is perceptually related, so in the considered case the content is as follows:

$$
P 1 T 1(a) \wedge P 2 T 2(b)
$$


According to (6), the experience is accurate if, and only if, the particular $a$ is in $P 1$ at $T 1$ and the particular $b$ is in $P 2$ at $T 2$. However, that is exactly what happens in the apparent motion case: the object $a$ is in $P 1$ at $T 1$ and the object $b$ is in $P 2$ at $T 2$. In consequence, using the particular notion of content produces an incorrect result, namely that the apparent motion illusion is an accurate experience.

Illusions of identity in which one perceives fewer objects than there really are also have static variants that do not involve perceiving objects at various moments. This can be demonstrated by investigating the amodal completion phenomenon (see Millar 2016 for other reasons why amodal completion is problematic for the particular notion of content). Let's consider a case in which one sees an obstacle, such that there is a part of an object sticking out from the left side of the obstacle and also a part of an object sticking out from the right side of the obstacle. If the edges of the left and the right parts stand in appropriate spatial relations, then both parts are experienced as fragments of a single object positioned behind the obstacle (Kimchi 2000). Of course, such an experience can be illusory because it may be that despite the arrangement of edges, there are two objects behind the obstacle: the object $a$ to which the left part belongs and the object $b$ which has the right part as its fragment.

Again, by using the general notion of content, the above illusion can be easily characterized as inaccurate. The content would be $\exists_{x, y} L P(x) \wedge R E L(x) \wedge R P(y) \wedge R E L(y) \wedge x=y$, where $L P$ designates the property of having the left part while $R P$ designates the property of having the right part. This content determines that the accuracy of the experience requires that the object having the left part is the same as the object having the right part. In consequence, the considered experience is inaccurate since, in fact, the left and right parts belong to separate objects. On the other hand, the particular view on content has a problem in obtaining this result. In the considered case, the particular content would be $L P(a) \wedge R P(b)$, because a subject is perceptually related to two particulars $a$ and $b$. However, this content exactly reflects the actual state of affairs, so it characterizes the illusion of identity as an accurate experience.

The same results are obtained if one considers the second type of illusion of identity, in which more objects are represented than there really are. Let's again start with a dynamic example concerning identifying objects in subsequent moments. It is well-established in vision science that human vision represents objects as being the same, as long as they move in a spatiotemporally continuous manner (Scholl 2007). However, if this constraint is broken, for instance an object suddenly disappears and reappears in some distant location, people have an experience as of numerically different objects. We may imagine a situation in which an object periodically becomes black and then returns to white while moving on a white background. In this case, when it turns white it blends with the background and ceases to be visible. As a result, a person has an experience as of several appearing and disappearing black objects. However, such an experience is illusory as, in fact, only one object is present.

Analogously as in the apparent motion illusion, the accuracy of this experience can be properly characterized using the general notion of content. Let's consider a case in which the object $a$ is visible in $P 1$ at $T 1$, then blends with the background, then is again visible in $P 2$ at $T 2$. In this situation, the experience has the following 
content ( $P 1 T 1$ designates the property of being in $P 1$ at $T 1$, while $P 2 T 2$ designates the property of being in $P 2$ at $T 2)$ :

$$
\exists_{x, y} P 1 T 1(x) \wedge R E L(x) \wedge P 2 T 2(y) \wedge R E L(y) \wedge x \neq y
$$

In consequence, the considered experience is inaccurate because (7) determines that the object in $P 1$ at $T 1$ should be different from the object in $P 2$ at $T 2$ while, in fact, there is only one object $a$.

On the other hand, the same conclusion cannot be reached using the particular notion of content. Due to the fact that both at T1 and T2 a subject is perceptually related to the object $a$, this object would be the only object that constitutes particular content:

$$
P 1 T 1(a) \wedge P 2 T 2(a)
$$

Such content entails that the considered illusion of identity is an accurate experience as (8) determines that the experience is accurate if, and only if, the object $a$ is in $P 1$ at $T 1$ and in $P 2$ at $T 2$. This is exactly what happens in the considered situation, as it involves only one object moving from $P 1$ to $P 2$.

The static variant of the illusion of identity, in which one represents more objects than there really are, can again be demonstrated by amodal completion. As in the previous example, let's assume that there is an obstacle with part of an object sticking out from its left side and part of an object sticking out from its right side. However, in this case, while the spatial relations between edges of the left and the right part do not cause an experience of a single object, in fact both parts are connected behind the obstacle and comprise the object $a$. The accuracy of this illusory experience can be properly characterized when the general view on content is adopted. The content would be $\exists_{x, y} L P(x) \wedge R E L(x) \wedge R P(y) \wedge R E L(y) \wedge x \neq y$, where $L P$ designates the property of having the left part while $R P$ designates the property of having the right part. Therefore, the experience is inaccurate, as the condition $x \neq y$ is not satisfied. On the other hand, the particular content will characterize this amodal completion illusion as an accurate experience. The particular content would be $L P(a) \wedge R P(a)$ and would entail that the considered illusion is accurate since, in fact, both parts belong to the object $a$.

The above considerations show that the particular notion of content has problems characterizing illusions of identity as inaccurate experiences, while these problems are not present in cases of the general content. Further below, I analyze possible ways in which a proponent of particular content can avoid the above difficulties and argue that each of these ways entails breaking one of the assumptions (A)-(C).

\section{Solutions for Particular Content}

To see whether a proponent of particular content can adequately characterize the accuracy of illusions of identity, let's focus on the apparent motion example, as it is the most common of such illusions and is experienced, for instance, each time we perceive moving objects on a computer screen. In the considered apparent motion example, a person has an experience as of only one object that moves 
from $P 1$ at $T 1$ to $P 2$ at $T 2$. In fact, the object at $T 1$ and the object at $T 2$ are two particulars, $a$ and $b$ respectively, so the experience is illusory. Nevertheless, the particular content is $P 1 T 1(a) \wedge P 2 T 2(b)$, because at each moment a subject is perceptually related to a different particular. This content determines that the experience is accurate if, and only if, object $a$ is in $P 1$ at $T 1$ and object $b$ is in $P 2$ at $T 2$. In consequence, the experience is characterized as accurate despite its illusory character.

One idea is to deny that the content contains two particulars. For instance, if the content were $P 1 T 1(c) \wedge P 2 T 2(c)$, the experience would be properly characterized as illusory. However, such a move explicitly violates assumption (B), namely that the objects needed for the accuracy of an experience are physical objects to which a subject is perceptually related, as $a$ and $b$ are only candidates for such objects in the considered situation. Furthermore, rejection of this assumption seems very implausible. To obtain content $P 1 T 1(c) \wedge P 2 T 2(c)$, the object $c$ that replaces $a$ and $b$ must be an object to which a subject is not perceptually related, or must be a non-physical, perhaps mental particular. Nevertheless, there is no clear criterion determining which of the objects that are not perceptually related to a subject should figure in content; in fact it seems generally unjustified to include such objects within content. On the other hand, treating object $c$ as a mental particular would lead to the conclusion that the particular notion of content entails a kind of sense-datum theory of perception. This is an unfavourable result, not only because sense-datum theories have serious drawbacks, but also because it betrays an important intuition motivating the particular notion of content, specifically that perception acquaints us with particular objects in the physical environment (Almäng 2013; Brewer 2015; Crane 2011).

Another variant of the above idea is to claim that in a case of apparent motion, no objects figure in content, so the considered experience has a gappy content $P 1 T 1\left(\_\right) \wedge P 2 T 2\left(\_\right)$. Of course, because apparent motion is a frequently occurring phenomenon, this violates assumption $(\mathrm{C})$, namely that common experiences do not have content with accuracy conditions that are impossible to satisfy. Furthermore, it seems unjustified to treat apparent motion as a kind of hallucination. The crucial characteristic of hallucinations involving object perception is that they are mental states in which one has an experience presenting objects but without standing in a proper perceptual relation to external particulars. However, in the case of apparent motion, the inaccuracy does not arise from the lack of perceptual relations, but because the identity of objects is recognized incorrectly.

What the above strategies have in common is that they try to solve the problem by denying that two different particulars figure in content. However, a different solution can be obtained by modifying not particulars but properties. It may be claimed that the particular content in a case of apparent motion contains two particulars, $a$ and $b$, but wrongly attributes to them the property of being identical. As a result, the content is as follows: $P 1 T 1(a) \wedge P 2 T 2(b) \wedge a=b$. The problem is that such content determines the accuracy conditions, which are self-contradictory, so that assumption (C), namely that common experiences do not have accuracy conditions that are impossible to satisfy, is violated, because the considered content entails that experience can be accurate only if there are two different objects, $a$ and $b$, that nevertheless are identical. Objects $a$ and $b$ are two different objects because they are exactly those 
objects to which a subject is perceptually related at $T 1$ and $T 2$. In a case of apparent motion these are distinct particulars.

Furthermore, the apparent motion phenomenon is an implausible candidate for having a self-contradictory content, not only because it is a common phenomenon, but also because such experiences present coherent states of affairs. They present that there is a single moving object; and such situations are certainly possible. Illusions that are likely to have self-contradictory accuracy conditions, like the waterfall illusion, are different in this respect, as they present that mutually exclusive properties are possessed by the same object.

A possible way out is to claim that contents such as $P 1 T 1(a) \wedge P 2 T 2(b) \wedge a=b$ are not self-contradictory because they can be interpreted as perceptual counterparts of Frege's puzzles about beliefs. For example, as famously proposed by Kripke (1979), we may imagine a person named Pierre who heard some stories about London and formed a belief Londres est jolie ("London is pretty" in French). Later, he learned English and started to live in London without knowing that Londres refers to this city. Because he lived in an unattractive part of the city, he formed a belief London is not pretty. It consequence, it may seem that he has contradictory beliefs. However, a popular response is that his beliefs are not contradictory because each belief refers to London under a different mode of presentation connected, for instance, with the circumstances in which the beliefs have been formed (see Bach 1997; Crimmins and Perry 1989; Salmon 1995).

One may propose that the same strategy can be applied in our perceptual example: it is not contradictory that particulars $a$ and $b$ are represented both as identical and as distinct, because in each case they are represented under a differing mode of presentation. However, such a move is not viable (similar arguments have been proposed earlier by Millar 2016, pp. 19-21). In the perceptual context, a statement that an element of content is represented under differing modes of presentation can mean two things: (a) that it is represented in phenomenally different ways or (b) that it is represented by using differing representational vehicles. Regarding the first option, a shape-property may be represented under differing phenomenal modes of presentation when it is represented both by vision and by touch. We may also imagine cases like those involving an inverted spectrum when the same colour property is first represented under a red mode of presentation, and then under a green mode (see Chalmers 2006). However, there is no such phenomenal variety in the considered example of apparent motion. It is simply not the case that each of the particulars $a$ and $b$ phenomenally looks in two differing ways during the apparent movement, and so there is no basis on which to postulate that each particular is represented under two phenomenal modes of presentation. According to the second option, a difference in perceptual modes of presentation is a difference in used representational vehicles. Within contemporary psychology, it is widely claimed that individual objects are represented by virtue of creating "object-files". Such files can be understood as mental devices containing information about the properties of a single object, and can be used to track how the object's properties change over time (see Kahneman et al. 1992 for a classical source). An object-file is created when an object is somehow perceptually selected, for instance by differentiating it from ground and focusing attention (Palmer and Rock 1994; Scholl 2001). It may happen that a single object 
is represented by two object-files corresponding to two modes of presentation. For instance, it may be the case that an object enters the visual field and an object-file is created for it. Then, it suddenly disappears, and subsequently appears again with some different properties, such that the visual system has to distinguish it from the ground for the second time. Due to lack of spatiotemporal continuity it is likely that visual mechanisms do not re-identify it as the same object, and therefore create a new object file instead of updating the earlier one (Scholl 2007). Nevertheless, it is implausible that an analogous situation happens in the apparent motion case. Each of the particulars $a$ and $b$ is distinguished from the ground only once, and there is no reason to postulate that the visual system creates two object-files for the particular $a$ and two object-files for the particular $b$. In fact, due to experienced spatiotemporal continuity, only one object-file is formed that incorrectly stores information about distinct objects.

The above problems with self-contradictory character of content could be amended by using a different predicate that ascribes something false to the considered objects. For instance, such a predicate could express not that $a$ is identical to $b$, but that $a$ is spatiotemporally continuous with $b$ or that $a$ moves from $P 1$ to $P 2$. However, this is not a viable solution as it misinterprets what happens in the apparent motion experiences. Such experiences not only present that an object moves from one place to another but also that through all moments of movement it is numerically the same object. This is a crucial feature of apparent motion illusion, which allows one to automatically treat objects displayed on a screen as remaining the same despite qualitative changes. In fact, the claim that apparent motion involves a representation of identity is also well confirmed by psychological results, as, according to them, in an ordinary situation, ${ }^{1}$ if an object $x$ is experienced as spatiotemporally continuous with an object $y$, then $x$ and $y$ are experienced as being numerically the same (see Scholl 2007 for a review).

Another variant of the above idea would be to claim that content in a case of apparent motion should not be characterized by a conjunctive statement such as $P 1 T 1(a) \wedge P 2 T 2(b)$. Instead, when experiencing apparent motion one has two accurate experiences with simple contents, $P 1 T 1(a)$ and $P 2 T 2(b)$, which jointly cause a third, inaccurate experience whose content describes a relation between objects $a$ and $b$. However, if this third, inaccurate experience does not attribute sameness to objects $a$ and $b$, then it has mischaracterized the apparent motion illusion. On the other hand, if sameness is attributed to these objects, then again assumption (C) is not satisfied.

The final solution available for the proponent of particular content is to reject assumption (A), namely that every element of content contributes to the determination of accuracy conditions. Without this assumption, the particulars $a$ and $b$ can still figure in the content $P 1 T 1(a) \wedge P 2 T 2(b)$, but they do not determine that the accuracy of an experience requires the presence of these two objects. However, it

\footnotetext{
${ }^{1}$ Exceptions may involve cases in which objects are experienced as dividing or merging, or when a task it too cognitively demanding (e.g., many objects has to be tracked simultaneously or objects move too fast).
} 
is not enough to leave the considered content as it is, and only exclude $a$ and $b$ from determining the accuracy conditions. In this case, the only elements of content that determine the accuracy conditions are predicates $P 1 T 1$ and $P 2 T 2$. In consequence, the considered apparent motion experience would be accurate if, and only if, the properties designated by $P 1 T 1$ and $P 2 T 2$ are instantiated. This condition is satisfied in the considered apparent motion experience, so again this experience would be mistakenly characterized as accurate.

If particular elements of content do not contribute to the determination of accuracy conditions, then content like $P 1 T 1(a) \wedge P 2 T 2(b)$ has to be modified by adding elements, which would guarantee that the apparent motion experience is illusory. These elements must determine that the accuracy of experience requires the presence of one object perceptually related to a subject, such that it in $P 1$ at $T 1$ and in $P 2$ at $T 2$. It is easy to see that in order to achieve this goal, the considered particular content must become very similar to general content like (5). More specifically, it would have the following form:

$$
\exists_{x, y} P 1 T 1(x) \wedge R E L(x) \wedge P 2 T 2(y) \wedge R E L(y) \wedge x=y \wedge x=a \wedge y=b
$$

In (9), the accuracy conditions are determined by the part of the content that does not contain particular elements, i.e., $\exists_{x, y} P 1 T 1(x) \wedge R E L(x) \wedge P 2 T 2(y) \wedge R E L(y) \wedge x=y$, while the part that contains particular elements, i.e., $x=a \wedge y=b$, has no influence on accuracy conditions. Overall, this content determines accuracy conditions in exactly the same way as in the case of general content: the experience is accurate if, and only if, there is an object in $P 1$ at $T 1$ perceptually related to a subject, and there is an object in $P 2$ at $T 2$ perceptually related to a subject, and the object in $P 1$ at $T 1$ is the same as the object in $P 2$ at $T 2$. However, the content also specifies that the object in $P 1$ at $T 1$ is the particular object $a$, while the object in $P 2$ at $T 2$ is the particular object $b$. Nevertheless, this part of the content does not influence the accuracy conditions. Thus, while the content is particular, since it contains particulars $a$ and $b$, it also properly characterizes the apparent motion experience as inaccurate.

Despite the fact that in the above "particularized general content" the particular elements do not play a role in determining the accuracy conditions, this does not necessarily mean that they are useless, or that postulating such content is unjustified. Current debates regarding perceptual content suggest at least two important functions that can be served by such particular elements. First, particular elements may allow for forming singular thoughts relying on perceptual experiences (Brewer 2015; Nanay 2012; Schellenberg 2016). Singular thoughts attribute properties to particular objects, so if they are based on perceptual experiences, the particular part of a "particularized general content" can determine the object that should be a referent of a singular thought. In this context, the lack of particular elements is considered a disadvantage of purely general contents, as it is unclear how such contents allow for determining the content of perception-based singular thoughts. Second, the particular elements may help to explain the particularity of perceptual phenomenology. It is believed that phenomenal character is closely connected to, or even supervenes on, representational content (see Byrne 2001; Davies 1992; Harman 1990 for classical discussions), and it seems intuitive that we are phenomenally presented with particular objects (Schellenberg 2016; 
Martin 2002). However, purely general contents seem to lack the resources needed to explain phenomenal particularity, as they do not contain a reference to any particular objects. On the other hand, the particular part of the "particularized general content" may amend this problem by determining that each experienced object is identical to a certain particular.

The above considerations suggest that the proponent of particular content cannot properly characterize the accuracy of illusions of identity without breaking one of the assumptions (A)-(C). The most promising way to do so is to abandon assumption (A) and claim that particular elements of content do not play a role in determining accuracy conditions. However, this solution comes at a price, as the particular notion of content has to borrow resources from the general notion. In consequence, a 'particularized general content' is obtained that is identical to a general content but with added components that identify experienced objects with particular entities. The accuracy conditions associated with such content are completely determined by its general part, and the particular part has to provide some other functions.

\section{Conclusions}

I have argued that both the general and the particular notion of content allow for the proper characterization of the accuracy of typical accurate perceptions, illusions, and hallucinations without breaking assumptions (A)-(C). These assumptions are: (A) all elements of content contribute to determining the accuracy conditions; (B) objects needed for accuracy are physical objects perceptually related to a subject; and (C) common experiences do not have accuracy conditions that cannot be satisfied. However, illusions of identity constitute an exception because they cannot be properly characterized as inaccurate using the particular notion of content without breaking one of the above assumptions. I have shown that the most promising move for proponents of particular content is to reject assumption (A). This leads to a notion of "particularized general content' whose general part determines the accuracy conditions, while its particular part serves other functions, for instance specifying particulars that are referents of singular thoughts.

Acknowledgements The work was supported by the National Science Center (Poland) Grant 2016/20/S/HS1/00090.

\section{Compliance with Ethical Standards}

Conflict of interest The author declares that he has no conflict of interest.

Ethical Statement I comply with the Ethical Standards of "Axiomathes".

Informed Consent and Animal Welfare The research did not involve human participants or animals.

Open Access This article is distributed under the terms of the Creative Commons Attribution 4.0 International License (http://creativecommons.org/licenses/by/4.0/), which permits unrestricted use, distribution, and reproduction in any medium, provided you give appropriate credit to the original author(s) and the source, provide a link to the Creative Commons license, and indicate if changes were made. 


\section{References}

Almäng J (2013) The causal self-referential theory of perception revisited. Dialectica 67(1):29-53

Bach K (1997) Do belief reports report beliefs? Pac Philos Q 78:215-241

Bach K (2007) Searle against the world: how can experiences find their objects? In: Tsohatzidis SL (ed) John's Searle's philosophy of language: force, meaning, and mind. Cambridge University Press, Cambridge, pp 64-78

Brewer B (2006) Perception and content. Eur J Philos 14(2):165-181

Brewer B (2015) The object view of perception. Topoi 36(2):215-227

Burge T (1991) Vision and intentional content. In: Lepore E, van Gulick R (eds) John Searle and his critics. Blackwell, Cambridge, pp 195-213

Byrne A (2001) Intentionalism defended. Philos Rev 110(2):199-240

Byrne A, Logue H (2008) Either/Or. In: Haddock A, McPherson F (eds) Disjunctivism. Perception, action, knowledge. Oxford University Press, Oxford, pp 57-94

Chalmers D (2006) Perception and the fall from Eden. In: Gendler TS, Hawthorne J (eds) Perceptual experience. Clarendon Press, Oxford, pp 49-125

Crane T (1988) The waterfall illusion. In: Gunther Y (ed) Essays on nonconceptual content. MIT Press, Cambridge, pp 231-236

Crane T (2011) The singularity of singular thought. Proc Aristot Soc Suppl 85:21-43

Crimmins M, Perry J (1989) The Prince and the phone booth: reporting puzzling beliefs. J Philos 86(12):685-711

Davies M (1992) Perceptual content and local supervenience. Proc Aristot Soc 92:21-45

Dokic J (1998) The ontology of perception: bipolarity and content. Erkenntnis 48:153-169

Genone J (2014) Appearance and illusion. Mind 123(490):339-376

Harman G (1990) The intrinsic quality of experience. Philos Perspect 4:31-52

Kahneman D, Treisman AM, Gibbs BJ (1992) The reviewing of object files: object-specific integration of information. Cogn Psychol 24(2):175-219

Kimchi R (2000) The perceptual organization of visual objects: a microgenetic analysis. Vis Res 40(10-12):1333-1347

Kriegel U (2009) Temporally token-reflexive experiences. Can J Philos 39(4):595-617

Kripke S (1979) A puzzle about belief. In: Margalit A (ed) Meaning and use, D. Reidel, Dordrecht, pp 239-283

Martin MGF (2002) Particular thought and singular thoughts. R Inst Philos Suppl 51:173-214

Matthen MP (2004) Features, places, and things: reflections on Austen Clark's theory of sentience. Philos Psychol 17(4):497-518

Millar B (2016) Frege's puzzle for perception. Research 93(2):368-392

Mulligan K (1999) Perception, particulars, and predicates. In: Fisette D (ed) Consciousness and intentionality: models and modalities of attribution. Springer, Dordrecht, pp 163-194

Nanay B (2012) Perceiving tropes. Erkenntnis 77:1-14

Palmer S, Rock I (1994) Rethinking perceptual organization: the role of uniform connectedness. Psychon Bull Rev 1(1):29-55

Pereira RHS (2016) Combining the representational and the relational view. Philos Stud 173:3255-3269

Prat OF (2006) Particularity and reflexivity in the intentional content of perception. Theoria 21(56):133-145

Salmon N (1995) Being of two minds: belief with doubt. Noûs 29(1):1-20

Schellenberg S (2010) The particularity and phenomenology of perceptual experiences. Philos Stud 149:19-48

Schellenberg S (2011) Perceptual content defended. Nous 45(4):711-750

Schellenberg S (2016) Perceptual particularity. Research 93(1):25-54

Scholl BJ (2001) Objects and attention: the state of art. Cognition 80:1-46

Scholl BJ (2007) Object persistence in philosophy and psychology. Mind Lang 22(5):563-591

Schroer R (2014) The goldilocks problem and the specificity of visual phenomenal content. Can J Philos 44(3-4):476-495

Searle J (1983) Intentionality: an essay in the philosophy of mind. Cambridge University Press, Cambridge

Siegel S (2010) The contents of visual experience. Oxford University Press, Oxford

Soteriou M (2000) The particularity of visual perception. Eur J Philos 8(2):173-189

Travis C (2014) The silence of the senses. Mind 113(449):57-94 